\title{
Influence of Typical Railway Objects in mmWave Propagation Channel
}

\author{
Danping He Member, IEEE, Bo Ai Senior Member, IEEE, Ke Guan Member, IEEE, Juan Moreno \\ García-Loygorri, Li Tian, Zhangdui Zhong Senior Member, IEEE, Andrej Hrovat Member, IEEE
}

\begin{abstract}
In the future railway services, wireless communication is the fundamental part and millimeter wave (mmWave) is foreseen to be a key enabler towards the smart railway. An accurate understanding of the propagation environment can assist designing both systems and railway infrastructures for better communication services. In this paper, the influence of typical objects to the mmWave propagation channel are analyzed for "Train-to-infrastructure" and "Intra-wagon" railway scenarios with various configurations. Propagation measurements are conducted in the mmWave band for the $\mathbf{1 2}$ most common railway materials. The corresponding electromagnetic parameters are obtained and a 3D ray tracing (RT) simulator is calibrated. The mean absolute error of the simulated S21 parameter is -53.5 dB, indicating that the calibrated RT can be used to generate the close-to-real mmWave channel for railway scenarios. Statistically consistent scenarios and deployments are generated, which enables drawing unbiased numerical results based on intensive RT simulations. The influence of typical objects and corresponding material compositions are then compared and significant objects are determined for each scenario. The results of this work not only imply how the propagation environment impacts on the propagation channel, but also makes suggestions to efficiently reconstruct railway environment models for an accurate RT based channel model. Moreover, the understanding of the influence of the environment at object and material levels will in turn guide the construction of railway infrastructure for better railway services.
\end{abstract}

Index Terms-Material characterization, millimeter wave, propagation channel, radio propagation measurement, railway communications, ray tracing simulation

\section{INTRODUCTION}

Due to the convenience and flexibility, more and more people prefer taking railway for traveling. According to statistics in 2016, more than $33 \%$ of passengers in Japan took railway each day [1] and the yearly ridership of urban railway and long-distance railway in China reached 16.1 billion and 2.8 billion, respectively. Besides, the Freight Delivery Metric (FDM) in the UK in 2016-2017 is higher than any previous

This work is supported by NSFC under Grant (61725101, 61771036, 61501021 and U1334202), Beijing Natural Science Foundation under Grant L161009, ZTE Corporation and State Key Lab of Rail Traffic Control and Safety Project under Grant (RCS2017ZZ004 and RCS2017ZT008).

Danping He, Bo Ai, Ke Guan and Zhangdui Zhong are with the State Key Laboratory of Rail Traffic Control and Safety, Beijing Jiaotong University, and also with Beijing Engineering Research Center of High-speed Railway Broadband Mobile Communications, China. (Corresponding author: Ke Guan, e-mail: kguan@bjtu.edu.cn)

Juan Moreno García-Loygorri is with Departamento de Teoría de la Señal y Comunicaciones, Universidad Politecnica de Madrid, Madrid, Spain

Li Tian is with ZTE Corporation, China

Andrej Hrovat is with Department of Communication Systems, Jožef Stefan Institute, Slovenia year [2]. The growing numbers not only bring pressures and challenges to the train operation and infrastructure construction, but also in turn promote the evolution and revolution of railway transport. In addition to traditional critical signaling, new functions such as efficient unmanned operations [3][7], onboard and wayside high definition video surveillance, Internet of Things for railways and onboard broadband internet service are desired to enable a safe, smart and comfort future railway transport.

One of the objectives of the fifth-generation (5G) mobile communications is to provide a similar user experience for end-users on the move as when they are static [8], [9]. In [10] and [11], at least five future railway service scenarios are defined, including "Train-to-infrastructure" (T2I), "Inter-wagon", "Intra-wagon", "Inside-the-station" and "Infrastructure-to-infrastructure" scenarios. Depending on the type of service, the estimated bandwidth requirements vary from $\mathrm{MHz}$ to $\mathrm{GHz}$. Therefore, in addition to the traditional sub-6 GHz bands, millimeter-wave (mmWave) band coping with multiple-input multiple-output (MIMO) technology is foreseen as a key enabler towards future railway broadband services. In 3GPP [12], [13], the $30 \mathrm{GHz}$ band has been proposed for T2I scenarios. In 2016, Horizon 2020 established 5GCHAMPION project [14] aiming to provide high-mobility broadband connections via $5 \mathrm{G}$ mmWave high capacity backhaul in $24 \mathrm{GHz}-28 \mathrm{GHz}$.

Before wireless communication system design and parameter setting, an accurate understanding of the propagation channel characteristics in spatial, time and frequency domains is important. Similar as vehicle-to-vehicle communication channels that have been thoroughly investigated for sub- $6 \mathrm{GHz}$ [15]-[17], there have been many studies on sub- $6 \mathrm{GHz}$ railway channels for various scenarios [18]-[24]. On the contrary, the mmWave band channel has been explored mainly for urban indoor and outdoor scenarios [25]-[30], and there are far less studies on mmWave channel for railway scenarios. The Japanese National Institute of Information and Communication Technology (NICT) is developing mmWave broadband T2I railway communication systems working at $40 \mathrm{GHz}$ and 90 $\mathrm{GHz}$ [31]. With 3GPP-like deployments, as shown in Fig. 1, the measured path losses of viaduct scenario are reported at the Asia-Pacific Telecommunity (APT) meeting [31]. The research on "Inter-wagon" communication is found in [32], which presents channel measurement for virtual coupling application at $60 \mathrm{GHz}$, where path loss and root-mean-square (RMS) delay spread are analyzed.

Due to the constraints on measurement equipment, instal- 


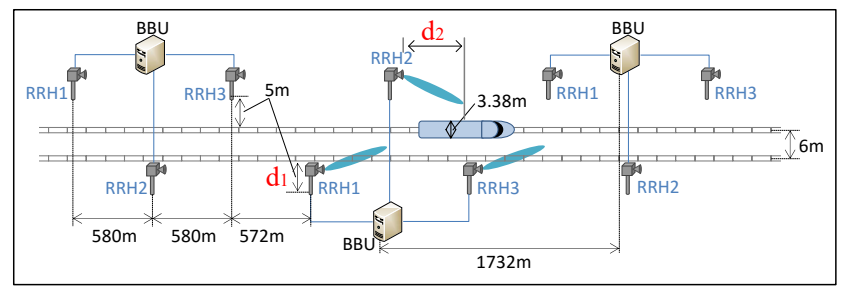

Figure 1. Deployment proposed by 3GPP [12], [13] for mmWave T2I railway communication

lations, workforce and time, mobile channel measurements encounter big challenges to acquire the aforementioned information for various complex scenarios and various speeds (max. $500 \mathrm{~km} / \mathrm{h}$ ). The authors of [28] and [29] combine limited mmWave channel measurements with extensive ray-tracing (RT) simulations to explore more characteristics, especially the angular characteristic for mmWave channel in urban outdoor scenarios. Furthermore, by taking advantage of the high spatial resolution of RT, both works in [33] and [34] propose new beamforming design technologies, which are verified in real trials. "Mobile Hotspot Network (MHN)" communication system is prototyped to support Gbps data rate services with speed over $400 \mathrm{~km} / \mathrm{h}$ [35]. Several trials have been done in Seoul subway rectangular tunnel with similar deployment as 3GPP proposal, while only signal-to-noise ratio and limited samples of channel impulse responses are recorded. More channel characteristics of the MHN system are compared in both circular and rectangular tunnel scenarios [36] via calibrated RT simulation. In [37], path loss and optimal antenna orientations are analyzed via RT simulation at $30 \mathrm{GHz}$ for a $3 \mathrm{GPP}-$ like deployment. As a result, RT simulations combined with a few measurements have been proved to be powerful in understanding the propagation channel.

However, it is known that the accuracy of RT mainly depends on the correct implementations of the propagation mechanism, environment model and antenna model, among which, reconstructing environment model and computation complexity are the two most time consuming part. The developments of high-performance computing and RT acceleration algorithms [38] have gradually reduced the time spent on tracing rays. Nevertheless, there is still a lack of quantitative analysis on the influence of the typical objects on the propagation channel to establish rules to model environment in an efficient way.

In this paper, the influence of typical objects is analyzed for mmWave channel in T2I and intra-wagon scenarios. The features of target environments are introduced. Typical objects, corresponding materials and geometries are summarized. Propagation measurements are conducted at the mmWave band for the most common materials on the typical objects. Electromagnetic (EM) parameters are extracted and input into RT, based on which, mmWave propagation mechanisms (direct, penetration, reflection, scattering and diffraction) are accurately simulated. Monte Carlo analysis approach is used to approximate unbias influence analysis via intensive RT simulations, and enormous statistically consistent environment models and deployments are generated for different scenarios based on the pre-defined variation domains. Significant objects and corresponding materials are determined through

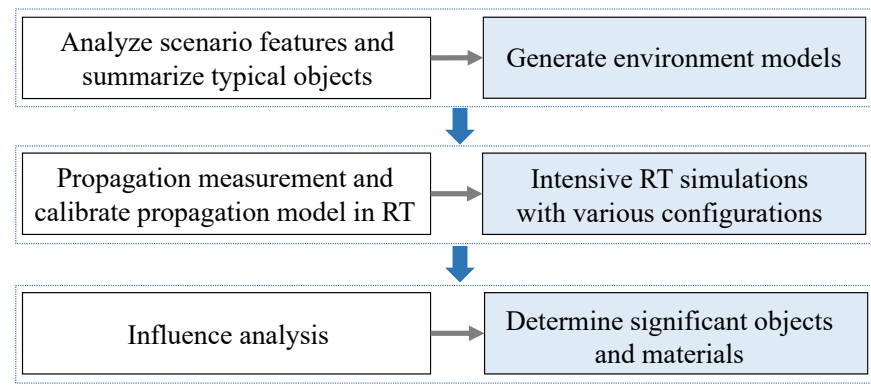

Figure 2. The framework of influence analysis

quantitative analysis of their influence. The results of this work not only imply how the propagation environment impacts on propagation channel, but also make suggestions to efficiently reconstruct railway environment models for accurate RT based channel modeling. Moreover, the understanding of the influence of the environment at object and material levels will in turn guide the construction of railway infrastructure for better railway services.

The remainder of this paper is organized as follows. The framework and preliminaries, including the features of railway scenarios, calibration of RT via propagation mechanism measurement, definitions and expressions, are introduced in Section II. The influence of typical objects in T2I and intrawagon scenarios are analyzed in Section III and Section IV, respectively. Conclusions are drawn in Section V.

\section{PROPOSED FRAMEWORK AND PRELIMINARIES}

The proposed framework of influence analysis is shown in Fig. 2. The work begins from analyzing scenario features and summarizing typical objects according to the code of design for railway infrastructure. Based on which, a large number of statistical consistent environment models are generated to draw unbias numerical results via Monte Carlo method. The three-dimensional (3D) ray tracer is calibrated via propagation measurement, and intensive close-to-real simulations are conducted with pre-defined configurations. The influence of typical objects and materials can be analyzed based on the extracted key parameters. T2I and intra-wagon scenarios are studied in this work.

\section{A. Features and typical objects in railway scenarios}

1) T2I scenario: Fig. 1 demonstrates T2I deployment at the mmWave band. The relay can be mounted on the top of the train, in the front/rear of train body or inside the driving cabin. Each baseband unit (BBU) is attached with three remote radio heads (RRHs). The RRH is linearly deployed along the two rails. The suggested distance from the RRH to the track is $5 \mathrm{~m}$. The antenna heights of both RRH and relay are almost equal. The suggested working frequency is $30 \mathrm{GHz}$ or $70 \mathrm{GHz}$ and the bandwidth is up to $1 \mathrm{GHz}$. The suggested distance between neighbor RRHs is $580 \mathrm{~m}$. MIMO systems with a unidirectional beam or bidirectional beam are recommended to compensate the high attenuation of mmWave band propagation.

The T2I communications could be in open-space scenarios (urban, rural, viaduct and cutting) and confined-space scenarios (tunnel) following the linear deployment proposal in 3GPP 
(Fig. 1). Some examples of the propagation environments are shown in Fig. 3. Although the construction of T2I scenarios varies, there are still many objects and corresponding materials in common.

In the open-space (urban, rural, viaduct, cutting) scenarios, train, tracks, catenary mast and ground are common objects. Train bodyshell (train body) and windows are made of metal and tempered glass (abbreviated to glass in the following descriptions), respectively. Tracks are composed of rails (made of metal), roadbed and sleepers. For current high-speed-train constructions, the roadbed and sleepers are usually made of concrete. The ground is usually flat and can be composed of cement, concrete or breakstones.

In urban, rural and viaduct, the sound barriers and buildings are often seen. The barriers, which are used in some regions to reduce noises, can be made of metal, polycarbonate (PLC plate) or concrete. Concrete, tempered glass, bricks, metal, etc., are often used to construct buildings. The density of buildings in the urban scenario is much higher than the rural. Since the track surface of the viaduct is tens of meters higher than the ground/water surface, the relative height of surrounding objects is smaller than that of urban flat ground, and some of them might be even below the track surface. Cutting is a man-made railway furniture, where the tracks flow at a lower level than its surroundings. The cuttings are usually made of concrete and stone with some vegetation on the surface.

Tunnels are classified as confined spaces, which have limited or restricted means of entry or exit. The shapes of tunnel cross-section can be either circular or rectangular. There are also some devices installed inside the tunnel for traffic monitoring and safety reasons, and they are usually made of metal and resins.

Table I lists the typical objects and corresponding materials in T2I scenarios. 8 structures, 12 types of objects and more than 9 materials are found. The geometry and location domains are provided according to statistical data of railway infrastructures. In summary, the tracks, catenary masts, ground are geometrical deterministic objects, barriers and cuttings are semi-deterministic objects in open space scenarios, as their geometries are usually determined whereas the existence and material compositions may vary slightly. The work of this paper considers the most common seen cuttings, which are higher than the train. The buildings along the track sides, which have varying geometries, material compositions, locations, etc., are named as random objects for open space scenarios. Tunnel wall and ceiling, ground, tracks and train are deterministic objects for tunnels, whereas the devices and wire cables are random objects.

2) Intra-wagon scenario: The purpose of intra-wagon communication is to establish the links between access points (APs) and user equipment (UE), which support onboard highdata-rate services. Fig. 4 shows an example of the deployment. The access points are usually installed on the ceiling or the wall of a wagon with variable azimuth locations. As users can access data services while sitting or standing, the height of UE varies. In the standing mode, line-of-sight (LOS) propagation is more likely to happen. Whereas, the seat backs block the
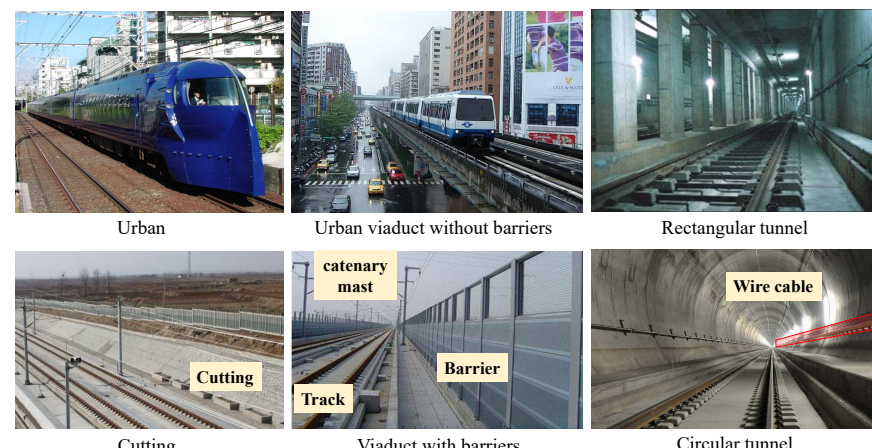

Rectangular tunne
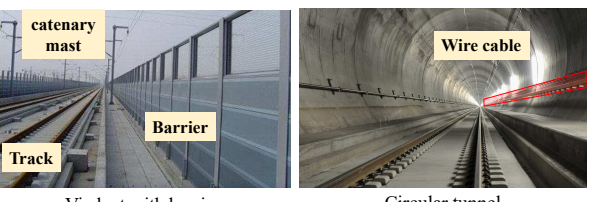

Viaduct with barriers

Circular tunnel

Figure 3. T2I scenarios

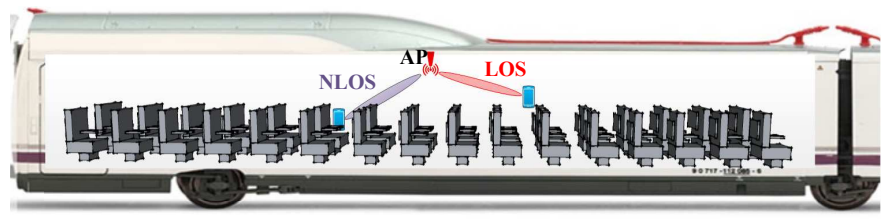

Figure 4. Intra-wagon deployment
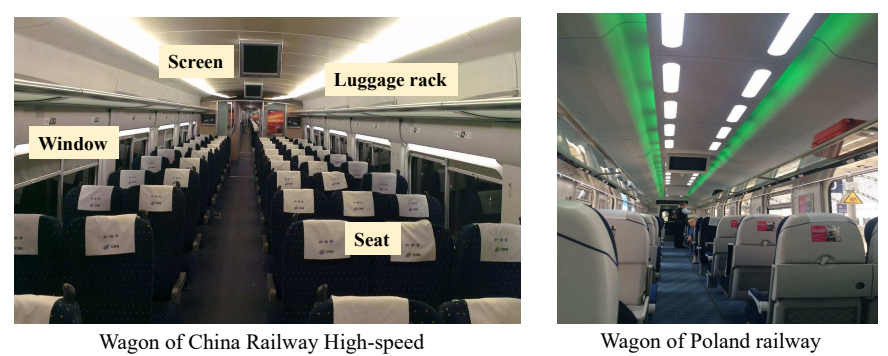

Wagon of Poland railway

Figure 5. Intra-wagon scenarios

direct path in the sitting mode. In the following analysis, the transmitter (Tx) is used to represent $\mathrm{RRH}$ and $\mathrm{AP}$, and receiver $(\mathrm{Rx})$ represents the relay and UE.

Fig. 5 shows some examples of the propagation environments inside the wagon. The seats, train body, windows, luggage racks and screens are the most common objects. Table II summarizes the objects, corresponding materials and geometries for intra-wagon scenarios, in which 3 typical structures, 7 parts and 6 types of materials are listed.

With the lists of objects and corresponding materials for the target scenarios, proper EM parameters can be obtained via dedicated propagation measurements to enable accurate RT simulations.

\section{B. The ray tracer and parameters of propagation models}

The developed 3D RT can trace direct, penetrated, reflected, scattered and diffracted paths. The EM computation is based on standardized or well known propagation models as 3GPP TR38.900 [39] and [40]. Since the EM parameters of a propagation model vary with material and frequency, the key towards accurate RT simulation for railway communication scenarios, is to obtain dedicated propagation parameters for the materials listed in Table I and Table II. Although in Rec. ITU-R P. 2040 [41] and Rec. ITU-R P.1238-7 [42], the dielectric parameters of concrete, glass and resin material can be obtained for $1 \mathrm{GHz}-100 \mathrm{GHz}$, they are mainly for urban and indoor environments. In addition, there is no derived relationship between the parameters and frequency. As the 
Table I

MAIN OBJECTS AND CORRESPONDING MATERIAL IN T2I SCENARIO

\begin{tabular}{|c|c|c|c|}
\hline Structure & Parts & Materials & Geometry \\
\hline \multirow[t]{2}{*}{ Train } & Wagon body & Metal & $\begin{array}{l}\text { W: }[3.2,3.3] \mathrm{m}, \mathrm{L}:[16,25] \mathrm{m}, \mathrm{H}:[3.7,4.27] \mathrm{m} \text {, } \\
\text { 8-16 wagons per train }\end{array}$ \\
\hline & Window & Tempered glass & L: $1.4 \mathrm{~m}, \mathrm{H}: 0.9 \mathrm{~m}$, Thickness: $3 \mathrm{~cm}-4 \mathrm{~cm}$ \\
\hline \multirow[t]{2}{*}{ Track } & Rail & Metal & $\begin{array}{l}\text { Distance between two rails (track width) } \mathrm{W}_{r}: 1.435 \mathrm{~m} \text {, } \\
\mathrm{H}: 15 \mathrm{~cm}\end{array}$ \\
\hline & Sleeper (and roadbed) & Concrete (high-speed) & $\begin{array}{l}\text { Concrete overall bed, } \\
\text { Inter distance between sleepers: }[0.5,0.6] \mathrm{m}\end{array}$ \\
\hline Ground & & Cement, Concrete, Soil, Breakstone & Flat \\
\hline \multirow{2}{*}{$\begin{array}{l}\text { Both sides of } \\
\text { the track }\end{array}$} & Catenary mast & Metal & $\mathrm{H}:[8,9] \mathrm{m}$ \\
\hline & Barrier & Metal, Polycarbonate, Concrete & H: $[5,7] \mathrm{m}$, distance to the center of track: $[1,8] \mathrm{m}$ \\
\hline \multirow{3}{*}{ Building } & Wall & Concrete, Glass, Brick, Metal & $\mathrm{W}:[10,100] \mathrm{m}, \mathrm{L}:[10,100] \mathrm{m}, \mathrm{H}:[5,80] \mathrm{m}$ \\
\hline & Window & Tempered glass & $\mathrm{W}:[1,2] \mathrm{m}, \mathrm{H}:[1,3] \mathrm{m}$ \\
\hline & Decoration & $\begin{array}{l}\text { Resin (Acrylic, Phenolic resin, } \\
\text { ABS Panel), Tiles (Ceramic Tile, } \\
\text { Marble, Granite), Metal }\end{array}$ & Area ratio (approximate): $0 \%-80 \%$ \\
\hline Billboard & & Metal, Resins & Pole H: $[18,24] \mathrm{m}$, board area $3 \mathrm{~W}^{2}, \mathrm{~W}:[6,8] \mathrm{m}$ \\
\hline Cutting & & Concrete, Stone, Vegetation & Inclination angle: $[35,40]$ degree, $\mathrm{H}:[6,14] \mathrm{m}$ \\
\hline \multirow{2}{*}{ Tunnel } & Wall and ceiling & Concrete & Cross section varies: rectangle and circle are typical shapes \\
\hline & Device & Metal, Resin & L: $[0.1$, tunnel length $] \mathrm{m}, \mathrm{W}:[0.1,0.5] \mathrm{m}, \mathrm{H}:[0.1,1] \mathrm{m}$ \\
\hline
\end{tabular}

Table II

MAIN OBJECTS AND CORRESPONDING MATERIALS IN INTRA-WAGON SCENARIO

\begin{tabular}{|l|l|l|l|}
\hline Objects & Parts & Materials & Geometry \\
\hline \multirow{2}{*}{ Wagon } & Wagon body & Metal, Resin & W: $[3.2,3.3] \mathrm{m}, \mathrm{L}:[16,25] \mathrm{m}, \mathrm{H}:[3.7,4.27] \mathrm{m}$ \\
\cline { 2 - 4 } & Window & Tempered glass & L: $1.4 \mathrm{~m}, \mathrm{H}: 0.9 \mathrm{~m}$, Thickness: $3 \mathrm{~cm}-4 \mathrm{~cm}$ \\
\hline \multirow{2}{*}{ Near the roof } & Screen & LCD Panel, Resin & W: $[0.5,0.8] \mathrm{m}, \mathrm{L}:[0.5,0.8] \mathrm{m}$ \\
\cline { 2 - 4 } & Luggage rack & Tempered glass & $\begin{array}{l}\text { W: }[0.5,0.6] \mathrm{m}, \mathrm{L}:[16,25] \mathrm{m}, \\
\text { Distance to the roof: }[0.5,0.6] \mathrm{m}\end{array}$ \\
\hline \multirow{3}{*}{ Seat } & Cushion \& back & Flannel fabric and Sponge with Resin & $\begin{array}{l}\text { Distance between back of seats: }[0.9,1] \mathrm{m} \\
\text { Num. of seats per row: 5, Number of rows per wagon: } 13-17 \\
\text { Aisle width } 0.85 \mathrm{~m}\end{array}$ \\
\cline { 2 - 4 } & Seat bracket & Resin & \multicolumn{2}{|c|}{} \\
\cline { 2 - 3 } & Armrest & Resin &
\end{tabular}

material parameters are frequency dependent, the provided parameters have limits for broadband RT simulations.

Therefore, dedicated propagation measurement is conducted for the aforementioned materials and objects. Fig. 6(a) shows the principle of propagation measurement for penetration, reflection and scattering. The $\mathrm{Tx}$, the $\mathrm{Rx}$ and the material are installed on a turning table. The material parameters at different incidence/arrival angles can be extracted via rotating the $\mathrm{Tx} / \mathrm{Rx} / \mathrm{material}$ in azimuth. The propagation measurement platform is shown in Fig. 6(b). A Keysight N5247A Vector Network Analyzer (VNA) is used to measure the $S_{21}$ parameter from $18 \mathrm{GHz}$ to $40 \mathrm{GHz}$. A spot focusing lens antenna is used at the Tx and a horn antenna is used at the Rx. As the focal spot diameter of the spot focusing lens antenna is 3 $\mathrm{mm}$ and the minimum side length of the measured object is 10 $\mathrm{cm}$, the influence of the surroundings are eliminated from the measurement. The motion controller is programmed to turn the incidence/arrival angle with a minimum resolution of $1^{\prime}$. Based on this principle, the 12 most common materials (Table I and Table II) are selected and measured, as shown in Fig. 7. The parameters of EM propagation models of penetration, reflection and scattering can be extracted and fitted for the materials. Afterwards, the fitted parameters are implemented in the material database of the RT.

Fig. 8(a) shows the scattering measurement result for concrete at $26 \mathrm{GHz}$, the scattering power increases as the scattering angle approaches the reflection angle, and the maximum power is achieved at the reflection direction. When the incidence angle is increased, the observed maximum scattering power increases as well. Fig. 8(b) compares the scattering powers between the fitting results and measured data. The

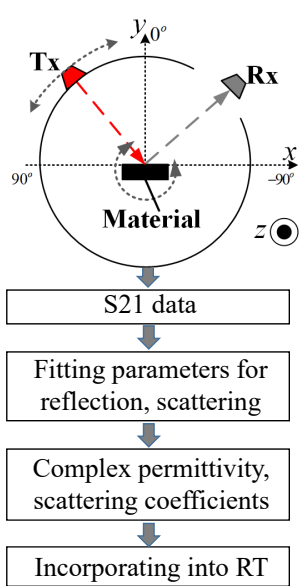

(a) Measurement principle

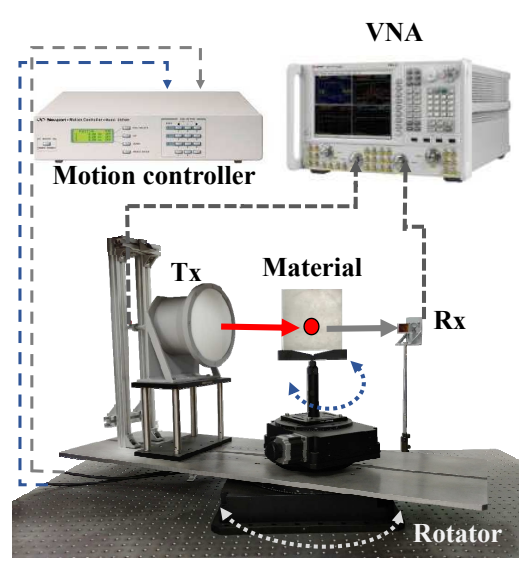

(b) Propagation measurement platform

Figure 6. Propagation mechanism measurement: principle and platform

directive scattering model [40] is considered in this work, and the frequency-dependent scattering coefficients are fitted accordingly for different materials. By comparing with the measurements ( $S_{21}$ parameters), the absolute errors of the fitting results of the directive scattering model are obtained. Fig. 9 shows the Cumulative Distribution Function (CDF) of the absolute fitting errors for the 12 materials within 18 GHz-40 GHz. The mean absolute error of the RT simulation results compared with the measured $\mathrm{S}_{21} \mathrm{~S}$ is $-53.5 \mathrm{~dB}$, the standard deviation of the error is $18.1 \mathrm{~dB}$ and the maximum error is $-23.4 \mathrm{~dB}$. The comparison implies that the RT can be used to conduct simulations for realistic radio propagation after incorporating the fitting parameters to the propagation models. Hence, key parameters can be extracted at ray level, 


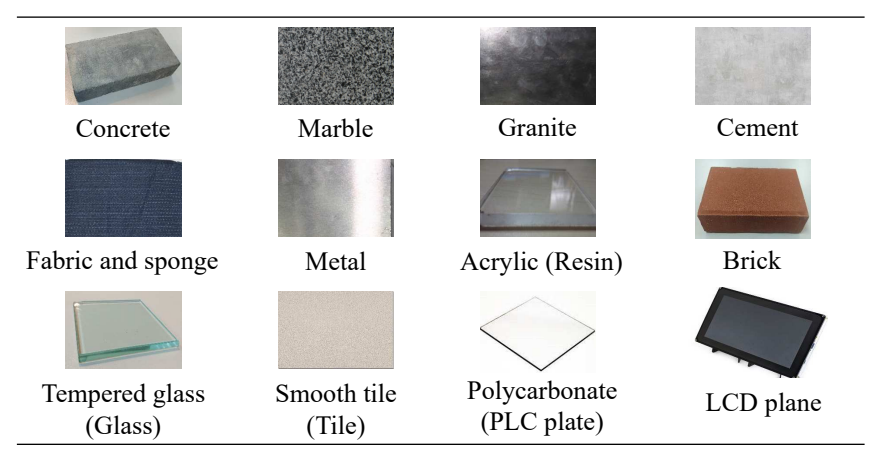

Figure 7. The 12 different analyzed materials considered in this work

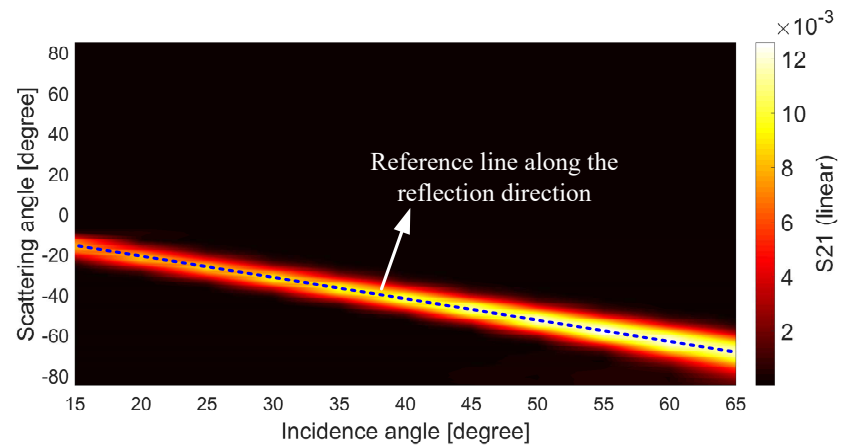

(a) Propagation measurement

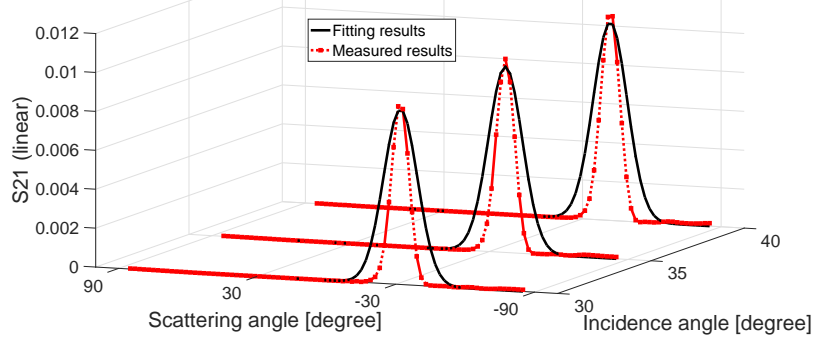

(b) Comparison between the fitting result and the measurement

Figure 8. Scattering measurement and the fitting result by the directive scattering model [40]: concrete, at $26 \mathrm{GHz}$

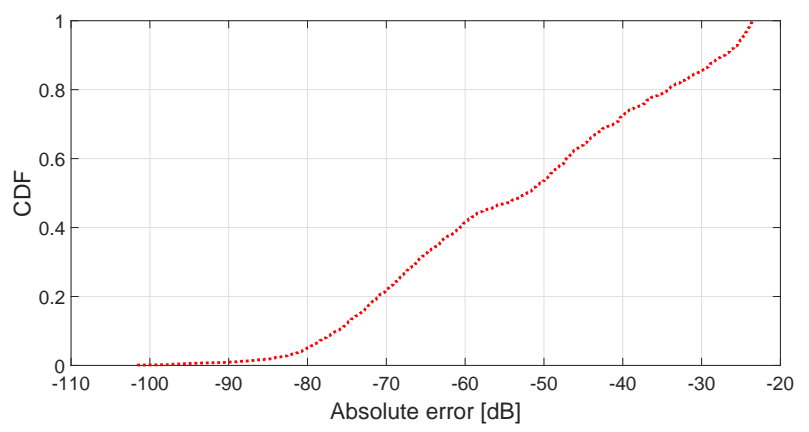

Figure 9. CDF of the absolute error of the fitting results for the 12 materials at $18-40 \mathrm{GHz}$

which enables detailed analysis of the influence of objects and materials.

\section{Preliminaries}

1) Simulation configurations: The simulation parameters are summarized in Table III. The Tx and $\mathrm{Rx}$ use omni- directional antennas with vertical polarization. The antenna gain is $0 \mathrm{dBi}$, and the transmission power is $0 \mathrm{dBm}$. The influence of typical railway objects is analyzed in the mmWave band (26 GHz-40 GHz). Four deployments (D1-D4) are considered for the T2I scenarios, the sitting mode and standing mode are considered for the Intra-wagon scenario. More details are introduced in the following sections. The LOS propagation, reflection, penetration with unlimited times, scattering and $1^{\text {st }}$ order diffraction are considered in all the scenarios, and the maximum bounces of reflection path $N_{B}$ varies in different scenarios. Due to the waveguide effect, the $N_{B}$ in the tunnel is 10 , which is the largest compared to other scenarios. The details of environment modeling are introduced case by case.

Table III

SIMULATION PARAMETERS

\begin{tabular}{|c|c|c|c|c|}
\hline Antenna type & \multicolumn{4}{|c|}{ Omni-directional, vertical polarization, $0 \mathrm{dBi}$} \\
\hline Frequency & \multicolumn{4}{|c|}{$26 \mathrm{GHz}, 30 \mathrm{GHz}$ and $40 \mathrm{GHz}$} \\
\hline $\begin{array}{l}\text { Transmitting } \\
\text { power }\end{array}$ & \multicolumn{4}{|c|}{$0 \mathrm{dBm}$} \\
\hline \multirow[b]{2}{*}{ Deployment } & \multirow{2}{*}{\multicolumn{2}{|c|}{$\begin{array}{l}\text { D1 (Tx: trackside-Rx: on top of the train), } \\
\text { D2 (Tx: over the track-Rx: on top of the } \\
\text { train), } \\
\text { D3 (Tx: trackside-Rx: inside the driving } \\
\text { cabin), } \\
\text { D4 (Tx: trackside-Rx: in front of the train) }\end{array}$}} & \multicolumn{2}{|c|}{ Intra-wagon } \\
\hline & & & $\begin{array}{l}\text { Sittin } \\
\text { Stand }\end{array}$ & $\begin{array}{l}\text { lode, } \\
\text { mode }\end{array}$ \\
\hline \multirow{6}{*}{ Ray types } & & $\begin{array}{c}\text { T2I } \\
\text { open space }\end{array}$ & $\begin{array}{c}\mathrm{T} 2 \mathrm{I} \\
\text { tunnel }\end{array}$ & $\begin{array}{l}\text { Intra- } \\
\text { wagon }\end{array}$ \\
\hline & LOS & $\sqrt{ }$ & $\sqrt{ }$ & $\sqrt{ }$ \\
\hline & $\begin{array}{l}\text { Reflection } \\
\text { bounces } N_{B}\end{array}$ & 2 & 10 & 5 \\
\hline & Penetration & $\sqrt{ }$ & $\sqrt{ }$ & $\sqrt{ }$ \\
\hline & Scattering & $\sqrt{ }$ & $\sqrt{ }$ & $\sqrt{ }$ \\
\hline & Diffraction & $\sqrt{ }$ & $\sqrt{ }$ & $\sqrt{ }$ \\
\hline
\end{tabular}

2) Definitions: In RT simulations, a pair of $\mathrm{Tx} / \mathrm{Rx}$ is simulated as a single snapshot with the predefined configurations. A simulation task for an environment model is composed of $N_{s}$ snapshots. For each snapshot $s$, the intrinsic results include the number of rays $N_{r}$, the type of each ray $T(s, j)$, bouncing times $B(s, j)$, hit objects $O(s, j)$, corresponding materials $M(s, j)$ and ray energy $E(s, j)$. The received power of the snapshot $s$ is expressed as:

$$
P_{r x}(s)=\left|\sum_{j=1}^{N_{r}} E(s, j)\right|^{2}
$$

The accumulated power of rays that hit object $o$ is:

$$
P(s, o)=\left|\sum_{j=1}^{N_{r}} C_{j} E(s, j)\right|^{2}
$$

where

$$
C_{j}=\left\{\begin{array}{lr}
1, & O(s, j)=o \\
0, & \text { else }
\end{array}\right.
$$

The power ratio $R(s, o)$ of an object $o$ is expressed as $P(s, o)$ over the received power $P_{r x}(s)$ of the same snapshot :

$$
R(s, o)=P(s, o) / P_{r x}(s)
$$

The influence of $o$ in the current simulation is defined as 
the mean value of the power ratios of all the snapshots $N_{s}$ :

$$
I_{o}=\frac{\sum_{s=1}^{N_{s}} R(s, o)}{N_{s}}
$$

Similarly, the influence of a type of material $m$ is obtained by replacing $o$ with $m$ in (2)-(5):

$$
I_{m}=\frac{\sum_{s=1}^{N_{s}} R(s, m)}{N_{s}}
$$

As the $R(s, o)$ and $R(s, m)$ range from 0 to 1 , the linear value of $I_{o}$ and $I_{m}$ are within $[0,1]$ as well.

\section{INFLUENCE ANALYSIS FOR T2I SCENARIOS}

As discussed before, the random objects in T2I scenarios have more diverse geometries and positions compared to the deterministic and semi-deterministic objects. Furthermore, typical T2I objects can be made of different and multiple materials with different composition ratios. In order to analyze their influence on propagation channel and draw numerical results without loss of generality, all the diverse situations should be ideally traversed, which will take infinite time. Therefore, Monte Carlo method is used in this work to realize stochastic simulation and approximate unbias analysis. The following procedure, coping with the features of the T2I scenarios, randomly generates statistically consistent T2I environment models and RT simulations configurations:

1) Define the deployment region of $\mathrm{Tx}$ and $\mathrm{Rx}$, randomly generate pairs of Tx and Rx.

2) Analyze the influence of deterministic and semideterministic objects. Construct the deterministic and semi-deterministic objects $O_{d}$. Based on the contributions to the propagation channel, select the significant ones $O_{d}^{\prime}$, which then perform as a foundation for further analysis.

3) Define material composition and area ratio for random objects. Generate $n_{e}$ environment models for random objects with random number, sizes, locations for each material composition. Therefore, if $k$ material compositions are considered, the total number of evaluated environment models is $N_{e}=k \times n_{e}$.

4) Perform RT simulations to the $N_{e}$ environment models with pre-defined deployment and simulation parameters.

5) Aggregate the results to perform the influence analysis.

Although the 3GPP deployment proposal (Fig. 1) provides fundamental guidelines, more diversities are considered in this work. As shown in Fig. 1, $d_{1}$ is the two-dimensional (2D) azimuth distance from the Tx to the closest track. For trackside communication, $d_{1}$ ranges from $0 \mathrm{~m}$ to $5 \mathrm{~m}$, which is shorter than the distance between the barrier and the track. For overthe-track communication, the $\mathrm{Tx}$ is mounted over the track with a certain height, and $d_{1}$ ranges from $0.5 \mathrm{~m}$ to $0.72 \mathrm{~m}$ (half of the track width $W_{r}$ ). The Rx can be deployed either inside the driving cabin, in front of or on the top of the train. Thus, the heights of the Tx and Rx which are nearly the same, vary within three levels: in the front of the train $[0.9,2] \mathrm{m}$, inside the driving cabin $[2,3.5] \mathrm{m}$ and on the top of the train $[3.7,4.5] \mathrm{m}$. When the $\mathrm{Rx}$ is on the top of the train,
Table IV

COMBINATION OF DIFFERENT MAJOR PLANE AND ATTACHED PLANES FOR

\begin{tabular}{|c|c|c|c|}
\hline Type & Main plane & Attached plane & Typical object \\
\hline \multirow{7}{*}{ Random } & \multirow{2}{*}{ Glass } & Metal & \multirow{2}{*}{$\begin{array}{l}\text { Office building, } \\
\text { hotel, etc. }\end{array}$} \\
\hline & & Resin & \\
\hline & \multirow{3}{*}{$\begin{array}{l}\text { Concrete, Brick, } \\
\text { Granite, Marble } \\
\text { Tile }\end{array}$} & Metal & \multirow{3}{*}{$\begin{array}{l}\text { Residential building, } \\
\text { office building, etc. }\end{array}$} \\
\hline & & Glass & \\
\hline & & Resin & \\
\hline & \multirow{2}{*}{ Metal } & Glass & \multirow{2}{*}{ Warehouse, bill board } \\
\hline & & Resin & \\
\hline \multirow{2}{*}{$\begin{array}{l}\text { Semi- } \\
\text { deterministic }\end{array}$} & Metal & Resin & \multirow{2}{*}{ Barrier } \\
\hline & Concrete & - & \\
\hline
\end{tabular}
T2I OPEN SPACE SCENARIOS

the distance of $\mathrm{Rx}$ to the front of the train varies within [0, $\left.L_{\text {wagon }} / 2\right] \mathrm{m}$, where $L_{\text {wagon }}$ is the length of a wagon. The distance between $\mathrm{Rx}$ and the sidewall of wagon varies within $\left[0, W_{\text {wagon }}\right]$ ( $W_{\text {wagon }}$ is the width of a wagon), which is not restricted in the middle of the wagon. The distance between the Tx and the Rx along the rail direction is defined as $d_{2}$. In this work, the considered range of $d_{2}$ is $[0,1732] \mathrm{m}$, which is also the inter-BBU distance in the $3 \mathrm{GPP}$ proposal.

\section{A. T2I open space scenarios}

The barrier, cutting, ground, track, catenary mast and train are the deterministic/semi-deterministic objects. As stated in Table I, objects such as barriers, buildings and billboards can be constituted with different materials. Thus, a main plane together with an attached plane are used to represent a composite plane of the semi-deterministic and random objects. The area ratio $A_{r}$ is defined as the ratio of the area of the attached plane to the area of the main plane. By changing the geometry, area ratio and the material type of the main plane and corresponding attached plane, the diverse properties can be represented. Table IV lists the combinations of materials for different major planes and attached planes of the barrier, building and billboard. In this work, $A_{r}$ increases from $1 \%$ to $81 \%$ with an increasing step of $10 \%$. The random objects are generated at both sides of tracks according to the geometrical parameters specified in Table I. The total number of random objects ranges from 4 to 50 in each environment model. Examples of the randomly generated T2I open space 3D environment models are shown in Fig. 10. The basic object modules are constructed by using Sketchup tool while the material and geometry properties can be modified by Matlab code.

1) Selection of the deterministic part: The influence of the deterministic candidate objects of urban, rural and viaduct scenarios are shown in Fig. 11. B represents barrier: B1 is made of concrete, B2 is made of metal with PLC plate in the upper part, B3 is made of metal with PLC plate in the middle; $\mathrm{G}$ stands for ground: $\mathrm{G} 1$ is made of concrete, $\mathrm{G} 2$ is cement, G3 is soil dry; Mast represents the catenary mast. The barrier and ground have significant influence for all the deployments. On the contrary, track and catenary mast have trivial influence for all the deployments. For the D2 deployment, where the Tx is over the track and the Rx is on top of the train, the concrete sleepers are within the reflection region of the propagation, and more significant rays are generated by the sleepers. Thus, the influence of the sleeper is greater than 0.8 in D2, whereas, 

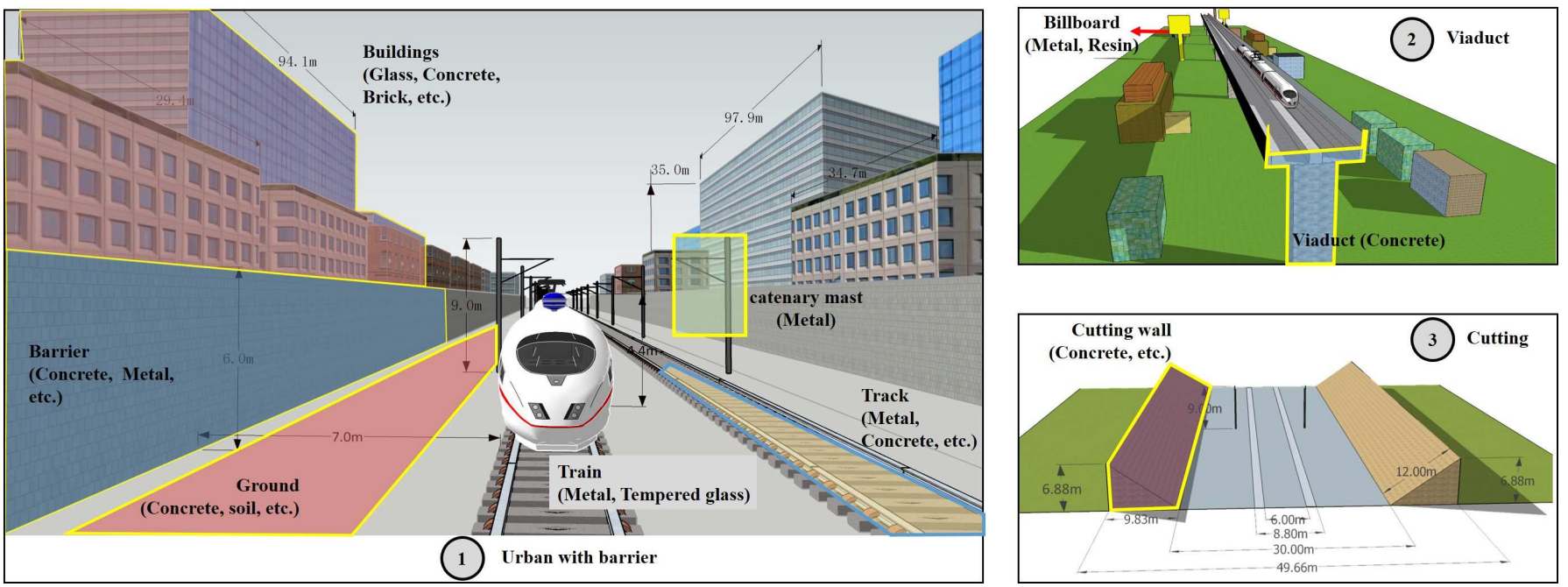

Figure 10. Examples of the generated 3D T2I open space environment models

its influence approximates 0 when the Tx is deployed at the trackside. Moreover, because both the $\mathrm{Tx}$ and the $\mathrm{Rx}$ are deployed high in D2, the reflected and significant scattering rays due to the barriers are mainly generated by the upper part. Due to the geometrical differences between the barriers and the sleepers, the influence of concrete barrier B1 is slightly less than the concrete sleeper. As the upper part of B2 is made of PLC plate and lower part is made of metal, the influence of $\mathrm{B} 2$ is higher than $\mathrm{B} 1$, and is almost identical to the sleeper. As the upper part of B3 is made of metal, the influence of $\mathrm{B} 3$ is the highest in D2. When the Rx is inside the driving cabin, the received rays penetrate the train window, thus the influence of the train is 1.0. However, when the $\mathrm{Rx}$ is on the top or in the front of the train, less rays hit the train before arriving at the $\mathrm{Rx}$, which makes the influence of the train negligible. The maximum influence of B2 is obtained in D4, in which the heights of Rx and Tx are smaller than the other cases and most of the rays hit on B2 are on the metal part rather than the PLC plate. When the height of Tx is within the middle of barrier (D3), rays of B3 are generated on the PLC plate, whereas in the other deployments, the intersected material is metal. As a result, the influence of B3 is the lowest in D3, compared with other deployments. The concrete (G1), cement (G2) and soil ground (G3) have very similar influence in the same deployment. When the Tx is at the trackside, the influence of ground is higher than D2 (over the track), and the value increases as the heights of Tx and Rx decrease. Fig. 12 shows how the influence of different objects varies as the frequency increases from $26 \mathrm{GHz}$ to $40 \mathrm{GHz}$. The influence of concrete barrier (B1) and ground (G1, G2 and G3) increases as frequency increases. The influence of catenary mast, train and B3 barely varies with frequency, the influence of B2 and sleeper decreases slightly $(<0.02)$ as frequency increases. As the maximum difference is 0.08 , the conclusions maintain the same from $26 \mathrm{GHz}$ to $40 \mathrm{GHz}$. Accordingly, the deterministic candidate objects of cutting scenario are evaluated at $26 \mathrm{GHz}$ (see Fig. 13). Due to the similar influence of different ground materials, G1 (concrete) is evaluated as a representative in this

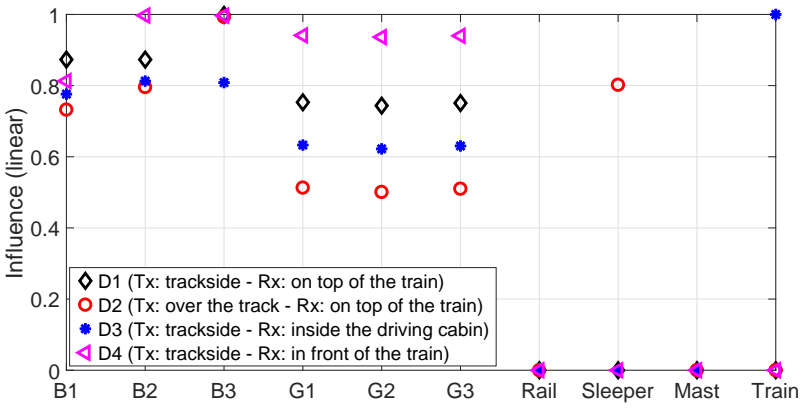

Figure 11. Influence of the deterministic candidate objects of urban, rural and viaduct scenarios at $26 \mathrm{GHz}$. B represents barrier: B1 is made of concrete, B2 is made of metal with PLC plate in the upper part, B3 is made of metal with PLC plate in the middle; G stands for ground: G1 is made of concrete, $\mathrm{G} 2$ is cement, G3 is soil dry

scenario. Because the cutting is inclined, far less reflected rays can arrive at $\mathrm{Rx}$ and the received scattering rays are far away from the center of scattering lobe, which make the influence of cutting much less important than the barrier. The influence of other objects are similar as in previous discussions.

The aforementioned results reveal that the track and catenary mast can be removed from the deterministic candidate list for all the deployment cases. The sleeper should be considered for D2, and removed for the rest cases. The train should be considered in D3 when the $\mathrm{Rx}$ is inside the driving cabin, and only the driving cabin should be modeled in this case. The cuttings are insignificant and can be excluded from the environment model. The barrier (if exists) and ground are significant and should be modeled in all the cases. Because the barriers don't always exist in open space scenarios, the study of the influence of the random objects will be further divided into "without barriers" and "with barriers" cases.

2) Without barriers: As discussed before, the plane of random objects are modeled as a main plane and an attached plane. To represent buildings and billboards, the material combination of both planes are summarized in Table IV. Fig. 14 shows an example of generated environment model for the Monte Carlo concept. The random planes (not cuboids, 


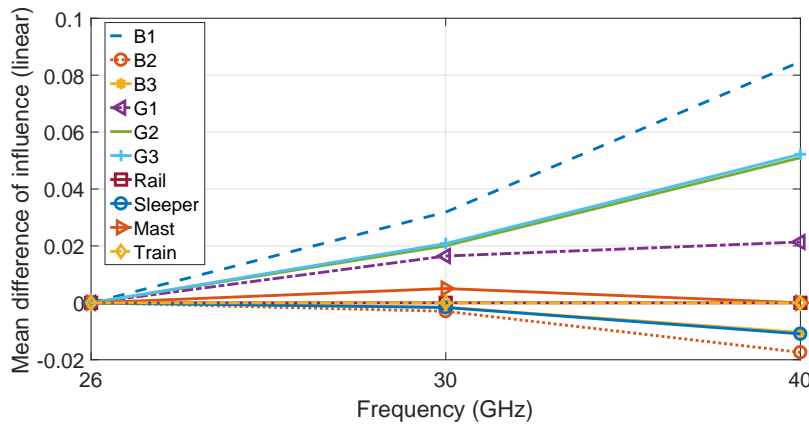

Figure 12. Differences of influence vary with frequency: the 10 deterministic candidate objects are evaluated with reference frequency at $26 \mathrm{GHz}$

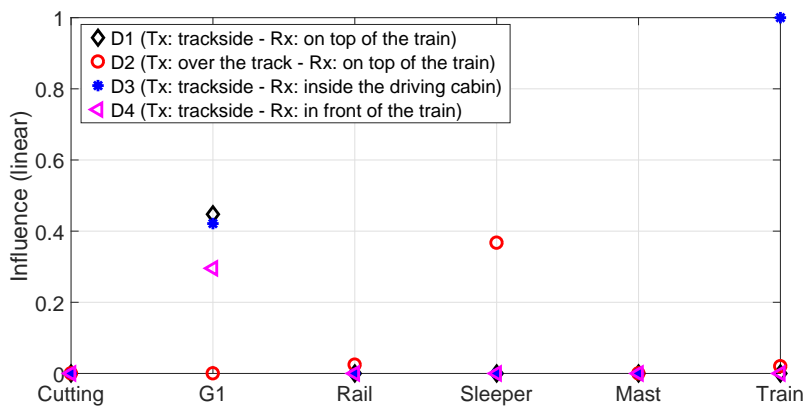

Figure 13. Average influence of the deterministic candidate objects in the cutting scenario at $26 \mathrm{GHz}$.

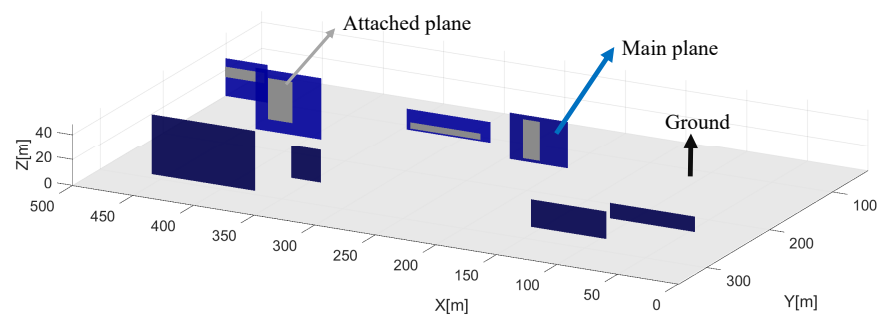

Figure 14. A demonstration of generating environment model for Monte Carlo evaluation: "without barriers" and area ratio is $45 \%$

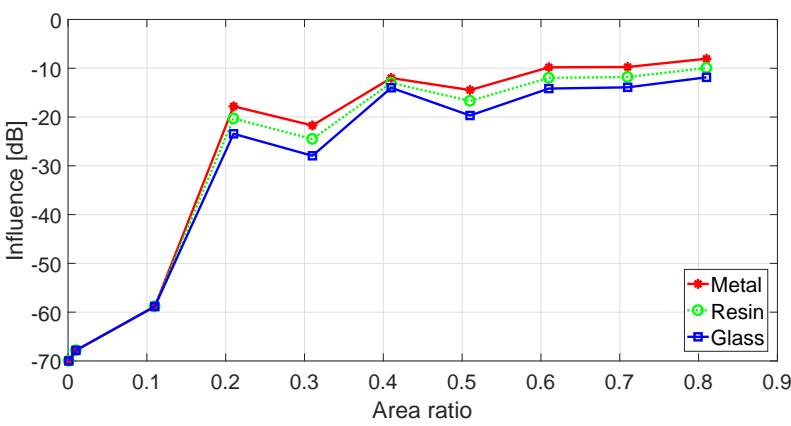

Figure 15. Influence of different material compositions in "without barriers": the comparison of the influence for the attached planes

for better demonstration), with different sizes, are distributed on both sides of the track. $n_{a}=1$ and the shapes of the attached plane are randomly generated with an area ratio of $45 \%$. Generally speaking, when the area ratio increases, the influence of the attached planes increases with fluctuation (see Fig. 15). When the area ratio is the same among all the attached materials, glass has the smallest influence, and

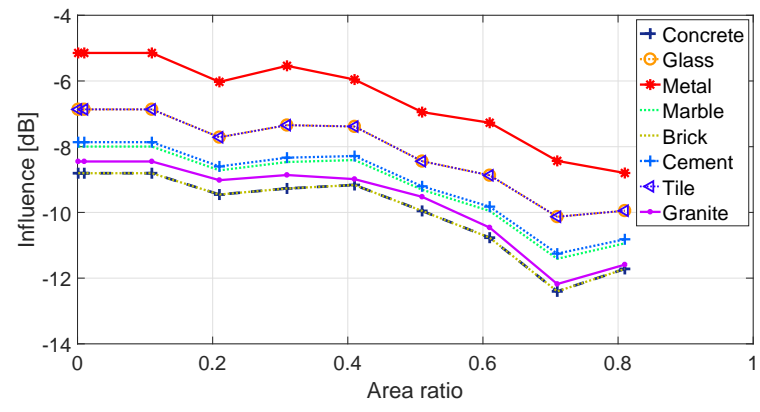

Figure 16. Influence of different material compositions in "without barriers": the comparison of the influence for the main planes

metal has the highest impact. However, the proportions of the attached planes can slightly differ in reality. For instance, the residential/office buildings have more than $25 \%$ of glass while the other materials are less than $2 \%$ each. As a result, the influence of glass can be at least $35 \mathrm{~dB}$ higher than resins and metal.

In the performance evaluation of communication systems, the channel impulses that are $25 \mathrm{~dB}-30 \mathrm{~dB}$ lower than the maximum value are usually not considered. If $-30 \mathrm{~dB}$ is selected as the threshold, the glass, resin and metal can be considered as significant materials when the area ratios of them are larger than $18 \%$.

Fig. 16 compares the influence of main plane materials. Concrete, brick, granite, marble, cement, tile, glass and metal, which are the fundamental materials of buildings, affect the propagation channel in ascending order. Besides, the influence of all the main planes is above $-13 \mathrm{~dB}$, and the value decreases slightly with the area ratio. As a result, objects that are within the evaluated range should be included in the environment model.

3) With barriers: Fig. 17 shows the CDF of the influence of the main planes when barriers exist along the trackside. The maximum influence is $-36 \mathrm{~dB}$ (metal) which is at least 24 $\mathrm{dB}$ lower than the minimum influence (concrete) in "without barriers". In this case, the average number of rays that hit random objects is 300 less compared to that of "without barriers". Therefore, the dominant rays with high power ratio are generated on the deterministic objects within the deterministic region when barriers exist, the random objects are insignificant and can be excluded from the environment model. Table V summarizes the significant deterministic and random objects in the open space scenarios.

\section{B. Tunnel scenario}

According to the previous discussion, the deterministic candidate objects include the tunnel wall and ceiling, ground, tracks and train. The random part includes the devices and wire cables, which are usually made of metal and resins and are installed on the tunnel wall. The $2 \mathrm{D}$ distance to the closest tunnel wall ranges from $0.05 \mathrm{~m}$ to $0.1 \mathrm{~m}$ and the tunnel length of generated model is $1000 \mathrm{~m}$. The objects are modeled as rectangle planes, and the area ranges from $0.1 \mathrm{~m}^{2}$ to $1000 \mathrm{~m}^{2}$ according to variation domains defined in Table I. Fig. 18 shows the reconstructed 3D tunnel models of rectangular (Type 


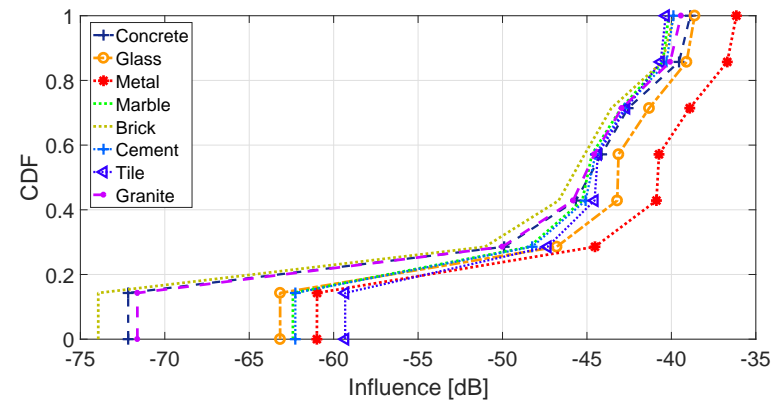

Figure 17. Influence of main planes in "with barriers"
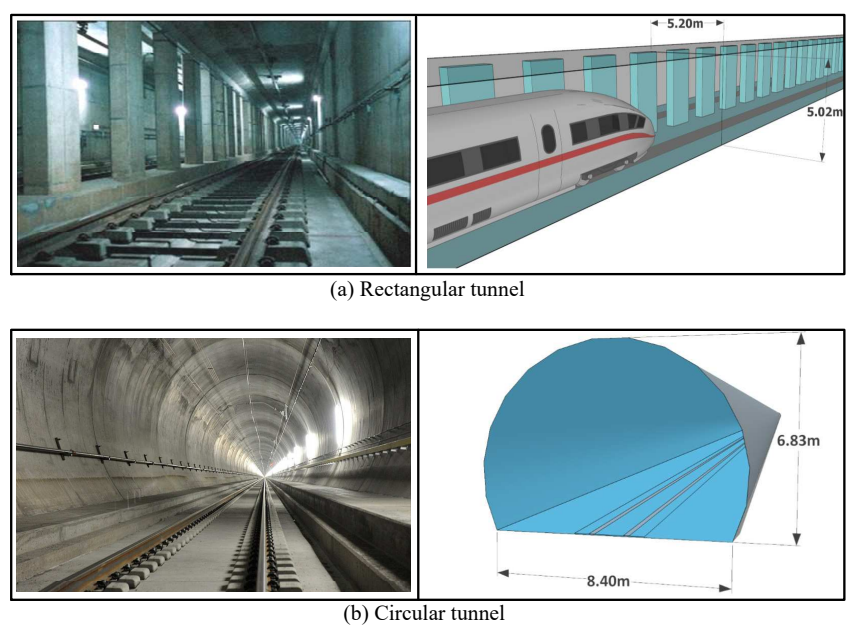

Figure 18. Reconstructed 3D tunnel models with different cross sections

I) and circular (Type II) shapes, and both of them are evaluated in this work.

1) Selection of the deterministic part: Fig. 19 compares the influence of deterministic candidates of the two types of tunnels. Tunnel is used to represent the tunnel wall, ceiling and ground. $I_{\text {tunnel }}>0.9$ in all the deployments in both tunnel types, thus the influence of tunnel is significant. The influence of sleeper $I_{\text {sleeper }}$ is 0.7 in D2 when the Tx is over the track, which is similar to the open space scenarios. Due to the waveguide effect, more rays are generated by the sleepers, thus the influence of sleeper $\left(I_{\text {sleeper }}=0.9\right)$ in D4 is much more significant than that in open space scenarios. In D1 and $\mathrm{D} 3$, the rays from the sleeper are blocked by the train, thus the $I_{\text {sleeper }}$ approximates 0 in both deployments. $I_{\text {train }}=1.0$ in D3 and the reason is the same as that in open space. Due to the geometrical difference between the two tunnel types, $I_{\text {train }}$ in D1 in Type II is significantly larger than that in Type I.

2) Influence analysis of the random part: The plane of the random part object is generated with a main plane and without attached plane, which is unlike the open space scenarios. Therefore, the area ratio is not applicable in this analysis. The absolute area of the object plane in square meter is used to evaluate the influence of objects quantitatively. Fig. 20 shows the variation of the influence with the area, metal and resin are compared for both tunnel types. Generally speaking, the influence of resin is slightly less than metal. The variation

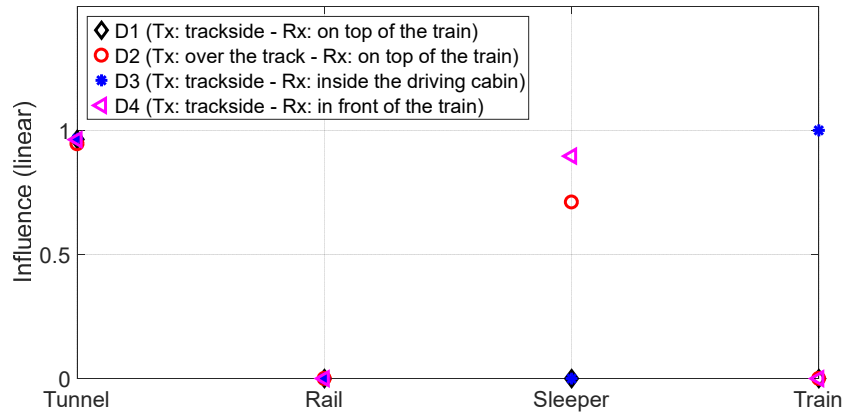

(a) Type I (rectangular) tunnel

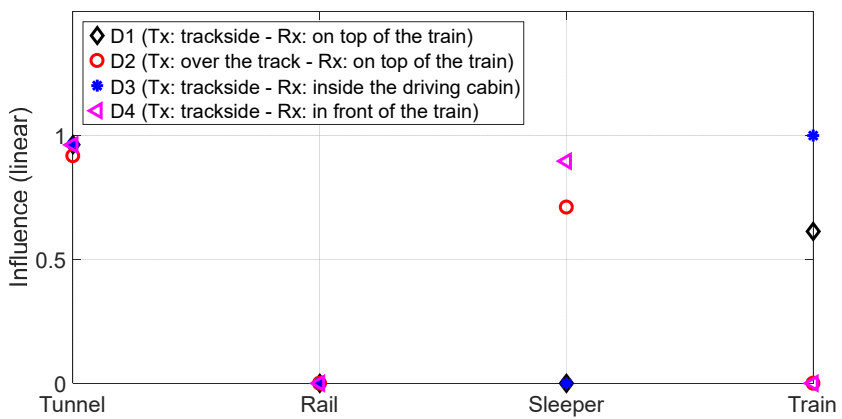

(b) Type II (circular) tunnel

Figure 19. Influence of deterministic objects of tunnel scenario at $26 \mathrm{GHz}$ : (a) Type I (rectangular) tunnel, (b) Type II (circular) tunnel

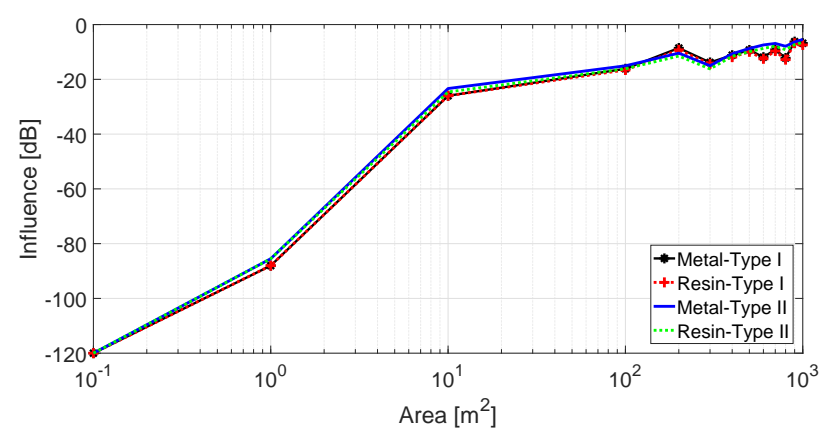

Figure 20. Influence comparison for random objects inside Type I (rectangular) and Type II (circular) tunnels

trends of both materials and numerical results in both scenarios are very similar: the influence grows dramatically when the area increases from $0.1 \mathrm{~m}^{2}$ to $10 \mathrm{~m}^{2}$; the value exceeds $-30 \mathrm{~dB}$ when the area is greater than $7 \mathrm{~m}^{2}$. As a result, the objects with areas larger than $7 \mathrm{~m}^{2}$ are significant and should be included in the tunnel environment model. The significant deterministic and random objects in tunnel scenario are summarized in Table V.

\section{INFLUENCE ANALYSIS FOR INTRA-WAGON SCENARIO}

Fig. 21 demonstrates the reconstructed wagon model, in which the typical objects listed in Table II are shown. The Tx is installed on the roof of the wagon, so users can access data service when sitting or standing. The two modes are evaluated by randomly placing the Rx inside the wagon, the $h_{R X}$ varies from $0.5 \mathrm{~m}$ to $0.8 \mathrm{~m}$ in the sitting mode, and $h_{R X}$ varies from $0.8 \mathrm{~m}$ to $1.6 \mathrm{~m}$ in the standing mode.

Fig. 22 compares the influence of different objects in the two modes. The wagon, window, screen and the seat cushion 


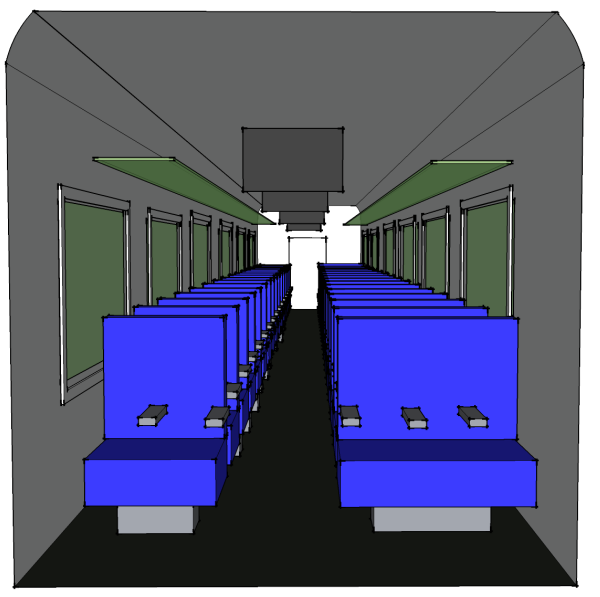

Figure 21. 3D model of the intra-wagon scenario

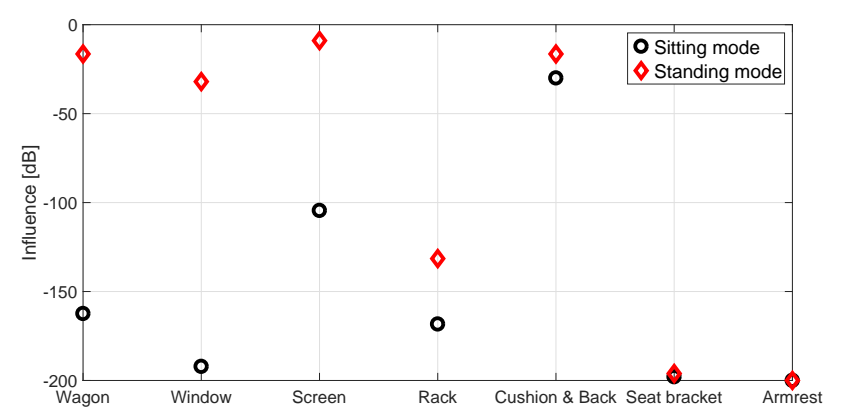

Figure 22. Influence comparison of typical objects for intra-wagon scenario

\& back have significant influence in the standing mode. As LOS path barely exists in the sitting mode, and many rays are blocked by the seats, the propagation condition is non-line-ofsight (NLOS). Only the seat cushion \& back are significant in the sitting mode. The influence of luggage rack, seat bracket and armrest are less than $-100 \mathrm{~dB}$ in both modes and are considered as insignificant objects. Table V summarizes the significant objects in this scenario.

\section{CONCLUSION}

In this paper, the influence of typical objects and materials in mmWave railway propagation environment are analyzed and compared. The features of "T2I" (urban, rural, viaduct, cutting and tunnel) and "Intra-wagon" scenarios with corresponding deployments are introduced. The environment models are divided into deterministic and random parts, and are reconstructed based on the code of design for railway infrastructure. Therefore, enormous virtually realistic environment models are generated to meet the requirements on drawing unbiased numerical results. Moreover, a 3D ray tracing simulator is calibrated via mmWave propagation measurement for 12 typical materials in railway scenarios. The average error of the $S_{21}$ is $-53.5 \mathrm{~dB}$, the standard deviation of the error is $18.1 \mathrm{~dB}$ with a maximum error of $-23.4 \mathrm{~dB}$. Thus, reliable simulations can be conducted and key parameters are extracted at the ray level. By taking this advantage, the influence of the objects and materials can be obtained. For T2I urban, rural and viaduct scenarios, the barrier, ground and train are significant deterministic objects.
When the barriers exist, the random part, which is outside of the deterministic region, are insignificant to the propagation channel. On the contrary, the random part is much more important if the barriers are absent: glass, resin and metal are significant decorated materials when their area ratios are larger than $18 \%$. Concrete, brick, granite, marble, cement, tile, glass and metal, which are the fundamental materials of buildings, affect the propagation channel in ascending order. For the T2I cutting scenario, the ground, sleeper and train are significant objects. For T2I tunnel scenario, circular and rectangular shapes are studied. The tunnel, sleeper and train are the common significant deterministic objects. A random object is significant in tunnel environment when its absolute area is larger than $7 \mathrm{~m}^{2}$. The sitting mode and the standing mode are studied for "Intra-wagon" scenario. In the sitting mode, only the seat cushion $\&$ back is significant. In the standing mode, the wagon, window, screen and the seat cushion \& back have significant influence. The results of this work not only imply how the propagation environment impacts on propagation channel, but also make suggestions to efficiently reconstruct railway environment models for accurate RT based channel modeling. Moreover, the understanding of the influence of the environment at object and material levels will in turn guide the construction of railway infrastructure for better railway services. In the future, more measurements and validations will be realized to improve this research work.

\section{REFERENCES}

[1] M. Thomas, "Research for tran commitee - the Japanese transport system," European Parliament.

[2] T. L. Gower, "Passenger and freight rail performance 2016-17 Q4 statistical release," Office of Rail and Road, May 2017.

[3] J. Kim, S. W. Choi, Y. S. Song et al., "Automatic train control over LTE: design and performance evaluation," IEEE Communications Magazine, vol. 53, no. 10 , pp. 102-109, Oct. 2015.

[4] A. Sniady and J. Soler, "LTE for railways: Impact on performance of ETCS railway signaling," IEEE Vehicular Technology Magazine, vol. 9, no. 2, pp. 69-77, Jun. 2014.

[5] O. H. Bettemir, "Detection of railway track from image by heuristic method," in 2015 23nd Signal Processing and Communications Applications Conference (SIU), May 2015, pp. 1366-1369.

[6] C. Sacchi and C. S. Regazzoni, "A distributed surveillance system for detection of abandoned objects in unmanned railway environments," IEEE Transactions on Vehicular Technology, vol. 49, no. 5, pp. 20132026, Sep. 2000.

[7] E. Páli, K. Máthé, L. Tamás et al., "Railway track following with the AR.Drone using vanishing point detection," in 2014 IEEE International Conference on Automation, Quality and Testing, Robotics, May 2014, pp. 1-6.

[8] "IMT vision - framework and overall objectives of the future development of IMT for 2020 and beyond," Recommendation ITU-R M.2083-0, Sep. 2015.

[9] C. X. Wang, F. Haider, X. Gao et al., "Cellular architecture and key technologies for 5G wireless communication networks," IEEE Соттиnications Magazine, vol. 52, no. 2, pp. 122-130, Feb. 2014.

[10] B. Ai, K. Guan, M. Rupp et al., "Future railway services-oriented mobile communications network," IEEE Communications Magazine, vol. 53, no. 10 , pp. $78-85$, Oct. 2015.

[11] K. Guan, G. Li, T. Kürner et al., "On millimeter wave and THz mobile radio channel for smart rail mobility," IEEE Transactions on Vehicular Technology, vol. 66, no. 7, pp. 5658-5674, Nov. 2016.

[12] Mitsubishi Electric and ETRI, "R1-165484 WF on evaluation assumption for high speed-train scenario 30GHz," 3rd Generation Partnership Project (3GPP) RAN1\#85, May 2016.

[13] Mitsubishi Electric, ETRI, and Ericsson, "R1-165926 WF on additional evaluation assumptions for high speed train scenario: Macro + relay around 30GHz," 3rd Generation Partnership Project (3GPP) RAN1\#85, May 2016. 
Table V

SUMMARIZATION OF SIGNIFICANT OBJECTS IN THE DISCUSSED SCENARIOS

\begin{tabular}{|c|c|c|c|}
\hline Scenario & Deployment & $\begin{array}{l}\text { Significant deterministic } \\
\text { objects }(>-30 \mathrm{~dB})\end{array}$ & Significant random objects/materials $(>-30 \mathrm{~dB})$ \\
\hline \multirow{4}{*}{$\begin{array}{l}\text { Open space } \\
\text { (urban, rural, viaduct) }\end{array}$} & T2I D1 & Barrier, ground & \multirow{4}{*}{$\begin{array}{l}\text { Without barriers: Buildings and billboards, decorated material: glass, resin and metal } \\
\text { with area ratios larger than } 18 \% \\
\text { With barriers: N/A }\end{array}$} \\
\hline & T2I D2 & Barrier, ground, sleeper & \\
\hline & T2I D3 & Barrier, ground, train & \\
\hline & T2I D4 & Barrier, ground & \\
\hline \multirow{4}{*}{$\begin{array}{l}\text { Open space } \\
\text { (cutting) }\end{array}$} & $\overline{\text { T2I D1 }}$ & Ground & \multirow{4}{*}{ N/A } \\
\hline & T2I D2 & Sleeper & \\
\hline & T2I D3 & Ground, train & \\
\hline & T2I D4 & Ground & \\
\hline \multirow{4}{*}{$\begin{array}{l}\text { Tunnel (rectangular, } \\
\text { circular) }\end{array}$} & T2I D1 & Tunnel, train (Circular only) & \multirow{4}{*}{ Resin and metal with absolute area larger than $7 \mathrm{~m}^{2}$} \\
\hline & T2I D2 & Tunnel, sleeper & \\
\hline & T2I D3 & Tunnel, train & \\
\hline & T2I D4 & Tunnel, sleeper & \\
\hline \multirow{2}{*}{ Intra wagon } & $\overline{\text { Sitting mode }}$ & Cushion \& back & $\overline{\mathrm{N} / \mathrm{A}}$ \\
\hline & Standing mode & $\begin{array}{l}\text { Wagon, window, screen, } \\
\text { cushion \& back }\end{array}$ & N/A \\
\hline
\end{tabular}

[14] E. C. Strinati, A. Clemente, J. Pajunpaa et al., "Deliverable D1.2: First period report," 5GCHAMPION Project, Jun. 2017. [Online]. Available: http://www.5g-champion.eu/Pages/DELIVERABLES.aspx

[15] A. F. Molisch, F. Tufvesson, J. Karedal et al., "A survey on vehicle-tovehicle propagation channels," IEEE Wireless Communications, vol. 16, no. 6, pp. 12-22, Dec. 2009.

[16] X. Cheng, Q. Yao, M. Wen et al., "Wideband channel modeling and intercarrier interference cancellation for vehicle-to-vehicle communication systems," IEEE Journal on Selected Areas in Communications, vol. 31, no. 9, pp. 434-448, Sep. 2013.

[17] X. Cheng, C. X. Wang, B. Ai et al., "Envelope level crossing rate and average fade duration of nonisotropic vehicle-to-vehicle ricean fading channels," IEEE Transactions on Intelligent Transportation Systems, vol. 15 , no. 1, pp. 62-72, Feb. 2014.

[18] C. X. Wang, A. Ghazal, B. Ai et al., "Channel measurements and models for high-speed train communication systems: A survey," IEEE Communications Surveys Tutorials, vol. 18, no. 2, pp. 974-987, Secondquarter 2016.

[19] A. Ghazal, C. X. Wang, B. Ai et al., "A nonstationary wideband mimo channel model for high-mobility intelligent transportation systems," IEEE Transactions on Intelligent Transportation Systems, vol. 16, no. 2, pp. 885-897, Apr. 2015.

[20] L. Zhang, J. Ding, B. Zhang et al., "Measurement and analysis of the broadband radio propagation in a high-speed railway station," in 2016 IEEE 83rd Vehicular Technology Conference (VTC Spring), May 2016, pp. $1-4$.

[21] C. Briso-Rodriguez, J. Cruz, and J. Alonso, "Measurements and modeling of distributed antenna systems in railway tunnels," IEEE Transactions on Vehicular Technology, vol. 56, no. 5, pp. 2870-2879, Sep. 2007.

[22] X. Yin, X. Cai, X. Cheng et al., "Empirical geometry-based randomcluster model for high-speed-train channels in umts networks," IEEE Transactions on Intelligent Transportation Systems, vol. 16, no. 5, pp. 2850-2861, Oct. 2015.

[23] B. Ai, R. He, Z. Zhong et al., "Radio wave propagation scene partitioning for high-speed rails," International Journal of Antennas and Propagation, vol. 2012, Jan. 2012.

[24] K. Guan, Z. Zhong, B. Ai et al., "Propagation measurements and analysis for train stations of high-speed railway at $930 \mathrm{MHz}$," IEEE Transactions on Vehicular Technology, vol. 63, no. 8, pp. 3499-3516, Oct. 2014.

[25] X. Wu, C. X. Wang, J. Sun et al., "60-GHz millimeter-wave channel measurements and modeling for indoor office environments," IEEE Transactions on Antennas and Propagation, vol. 65, no. 4, pp. 1912 1924, Apr. 2017.

[26] W. Fan, I. Carton, J. Ø. Nielsen et al., "Measured wideband characteristics of indoor channels at centimetric and millimetric bands," EURASIP Journal on Wireless Communications and Networking, vol. 2016, no. 1, p. 58 , Feb. 2016.

[27] B. Ai, K. Guan, R. He et al., "On indoor millimeter wave massive mimo channels: Measurement and simulation," IEEE Journal on Selected Areas in Communications, vol. 35, no. 7, pp. 1678-1690, Jul. 2017

[28] M. K. Samimi and T. S. Rappaport, "3-D millimeter-wave statistical channel model for 5G wireless system design," IEEE Transactions on Microwave Theory and Techniques, vol. 64, no. 7, pp. 2207-2225, Jul. 2016.
[29] S. Hur, S. Baek, B. Kim et al., "Proposal on millimeter-wave channel modeling for 5G cellular system," IEEE Journal of Selected Topics in Signal Processing, vol. 10, no. 3, pp. 454-469, Apr. 2016.

[30] J. Zhang, P. Tang, L. Tian et al., "6-100 GHz research progress and challenges from a channel perspective for fifth generation (5G) and future wireless communication," Science China Information Sciences, vol. 60, no. 8, pp. 1-16, Aug. 2017.

[31] T. Kawanishi, A. Kanno, H. Ogawa et al., "Proposal of a new working document of a draft new apt report on millimeter-wave band railway radiocommunication systems between train and trackside, and its work plan," in the 20th meeting of the APT Wireless Group (AWG-20), Sep. 2016.

[32] K. Yang, M. Berbineau, J. P. Ghys et al., "Propagation measurements with regional train at $60 \mathrm{GHz}$ for virtual coupling application," in 2017 11th European Conference on Antennas and Propagation (EUCAP), Mar. 2017, pp. 126-130.

[33] V. Degli-Esposti, F. Fuschini, E. M. Vitucci et al., "Ray-tracing-based mm-wave beamforming assessment," IEEE Access, vol. 2, pp. 13141325, Oct. 2014.

[34] Y. Yang, E. Mellios, M. Beach et al., "Evaluation of three-element MIMO access points based on measurements and ray tracing models," in 2015 IEEE 26th Annual International Symposium on Personal, Indoor, and Mobile Radio Communications (PIMRC), Aug. 2015, pp. 419-424.

[35] J. Kim and I. G. Kim, "Distributed antenna system-based millimeterwave mobile broadband communication system for high speed trains," in 2013 International Conference on ICT Convergence (ICTC), Oct. 2013, pp. 218-222.

[36] G. Li, B. Ai, K. Guan et al., "Channel characterization for mobile hotspot network in subway tunnels at $30 \mathrm{GHz}$ band," in 2016 IEEE 83rd Vehicular Technology Conference (VTC Spring), May 2016, pp. $1-5$.

[37] D. He, J. Yang, K. Guan et al., "Ray-tracing simulation and analysis of propagation for 3GPP high speed scenarios," in 2017 11th European Conference on Antennas and Propagation (EUCAP), Mar. 2017, pp. 2890-2894.

[38] L. Wang, K. Guan, B. Ai et al., "An accelerated algorithm for ray tracing simulation based on high-performance computation," in 2016 11th International Symposium on Antennas, Propagation and EM Theory (ISAPE), Oct. 2016, pp. 512-515.

[39] "Channel model for frequency spectrum above $6 \mathrm{GHz}$ (Release 14)," 3rd Generation Partnership Project (3GPP) TR 38.900-14.3.1, Jul. 2017.

[40] V. Degli-Esposti, F. Fuschini, E. M. Vitucci et al., "Measurement and modelling of scattering from buildings," IEEE Transactions on Antennas and Propagation, vol. 55, no. 1, pp. 143-153, Jan. 2007.

[41] "Effects of building materials and structures on radiowave propagation above about $100 \mathrm{MHz}$," Recommendation ITU-R P.2040-1, Jul. 2015.

[42] "Propagation data and prediction methods for the planning of indoor radiocommunication systems and radio local area networks in the frequency range $900 \mathrm{MHz}$ to $100 \mathrm{GHz}$," Recommendation ITU-R P.12387, Feb. 2012. 


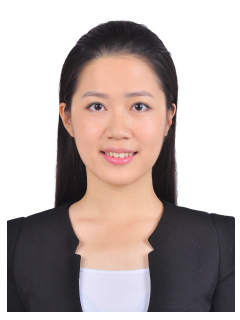

Danping He (M'16) received B.E.degree from Huazhong University of Science and Technology in 2008, M.Sc. degree from the Universite Catholique de Louvain (UCL) and Politecnico di Torino (PdT) in 2010, and Ph.D. degree from Universidad Politécnica de Madrid in 2014. She works in Huawei Technologies from 2014 to 2015 as a research engineer, and she is currently conducting postdoctoral research in the State Key Laboratory of Rail Traffic Control and Safety, Beijing Jiaotong University. She has authored/co-authored over 15 papers and 3 patents. Her paper received the best paper award in JCE 2013. Her current research interests include radio propagation and channel modeling, ray tracing simulator development and wireless communication algorithm design.

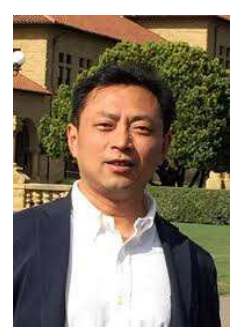

Bo Ai (M'00-SM'10) received his Master and Ph.D. degree from Xidian University in 2002 and 2004 in China, respectively. He graduated in 2007 with great honors of Excellent Postdoctoral Research Fellow in Tsinghua University. He is now working in Beijing Jiaotong University as a professor and advisor of $\mathrm{Ph} . \mathrm{D}$. candidates. He is a deputy director of State Key Lab of Rail Traffic Control and Safety. He is an associate editor for IEEE Trans. on Consumer Electronics and an editorial committee member of journal of Wireless Personal Communications.

He has authored/co-authored 6 books, 140 scientific research papers and 26 invention patents in his research area till now. His current interests are the research and applications OFDM techniques, HPA linearization techniques, radio propagation and channel modeling, GSM for railway systems, and LTE for railway systems. He is an IET Fellow and an IEEE Senior member.

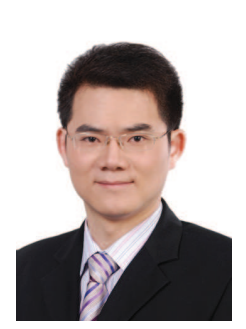

Ke Guan (S'10-M'13) received B.E. degree and $\mathrm{Ph} . \mathrm{D}$. degree from Beijing Jiaotong University in 2006 and 2014, respectively. He is an Associate Professor in State Key Laboratory of Rail Traffic Control and Safety \& School of Electronic and Information Engineering, Beijing Jiaotong University. In 2015, he has been awarded a Humboldt Research Fellowship for Postdoctoral Researchers. He was the recipient of a 2014 International Union of Radio Science (URSI) Young Scientist Award. His papers received 6 Best Paper Awards. In 2009, he was a visiting scholar in Universidad Politécnica de Madrid, Spain. From 2011 to 2013, he has been a research scholar at the Institut für Nachrichtentechnik (IfN) at Technische Universität Braunschweig, Germany. From September 2013 to January 2014, he was invited to conduct joint research in Universidad Politécnica de Madrid, Spain. His current research interests are in the field of measurement and modeling of wireless propagation channels, high-speed railway communications, vehicle-to- $\mathrm{x}$ channel characterization, and indoor channel characterization for high-speed short-range systems including future terahertz communication systems.

He has authored one book, two book chapters, more than 160 journal and conference papers, and one patent. He received the Huawei Excellent Student Award of China in 2013 and the First National Scholarship for Ph. D Candidates in 2012. He serves as a Publicity Chair in PIMRC 2016, the Session Convener of EuCAP 2015, 2016, and 2017, and a TPC Member for many IEEE conferences, such as Globecom, ICC and VTC. He has been a member of the IC1004 and CA15104 initiatives.

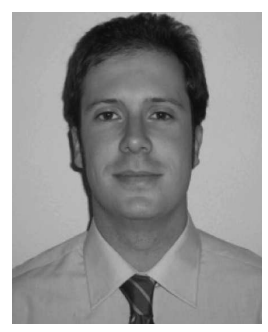

Juan Moreno García-Loygorri is a rolling stock engineer in the Engineering and Research Department of Madrid Metro, where he has leaded many projects on railway communications. He is also a part-time professor in the Universidad Politécnica de Madrid. He has been working in railways since 2007, first on high-speed and then in subways. He has participated in many railway-related research projects like Roll2Rail and Tecrail, and has authored more than 20 papers on railway communications. His research interests are channel measurement \& modelling, railway communications systems and software-defined radio. His $\mathrm{PhD}$ Thesis, presented in November 2015, was also focused on railway communications.

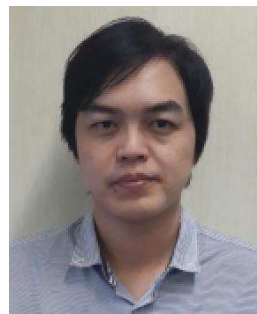

Li Tian received the bachelor degree in communication engineering and the Ph.D degree in Control Science and Control Engineering from Tongji University, Shanghai, China, in July 2009 and January 2015, respectively. From 2013 to 2014, he was a visiting Ph.D student at the Department of Electronics and Information Systems (DEIS), University of Bologna, working with Prof. Vittorio Degli-Esposti. $\mathrm{He}$ participated in the $5 \mathrm{G}$ project sponsored by National Natural Science Foundation of China. He is now a Senior Engineer at the Department of Algorithms, ZTE Corporation. His current research interests are in the field of $5 \mathrm{G}$ channel modeling and new air-interface. Dr. Tian serves as reviewer for a number of international journals including IEEE Transactions on Antennas and Propagation, IEEE Transactions on Vehicular Technology, IEEE Access, IEEE Antennas and Wireless Propagation Letters, and International Journal of Antennas and Propagation.

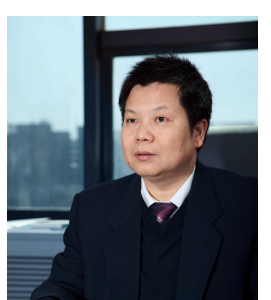

Zhangdui Zhong (SM'16) is a professor and advisor of Ph.D. candidates in Beijing Jiaotong University. He is now a director of School of Computer and Information Technology and a Chief Scientist of State Key Laboratory of Rail Traffic Control and Safety in Beijing Jiaotong University. $\mathrm{He}$ is also a director of the Innovative Research Team of Ministry of Education, and a Chief Scientist of Ministry of Railways in China. He is an executive council member of Radio Association of China, and a deputy director of Radio Association of Beijing. His interests are wireless communications for railways, control theory and techniques for railways, and GSM-R system. His research has been widely used in the railway engineering, such as Qinghai-Xizang railway, DatongQinhuangdao Heavy Haul railway, and many high-speed railway lines of China.

He has authored/co-authored 7 books, 5 invention patents, and over 200 scientific research papers in his research area. He received MaoYiSheng Scientific Award of China, ZhanTianYou Railway Honorary Award of China, and Top 10 Science/Technology Achievements Award of Chinese Universities.

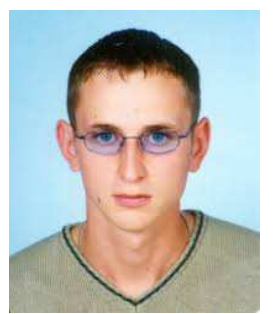

Andrej Hrovat (M'13) was born in Novo mesto, Slovenia, in 1979. He received a B.Sc. and M.SC. in Electrical Engineering from the University of Ljubljana, Slovenia, in 2004 and 2008, respectively. $\mathrm{He}$ obtained a Ph.D. degree in Electrical Engineering from the Joef Stefan International Postgraduate School, Slovenia, in 2011. He is currently a researcher in the Department of Communication Systems of the Joef Stefan Institute and assistant at the Joef Stefan International Postgraduate School. His research interests include radio signal propagation, channel modeling, terrestrial and satellite fixed and mobile wireless communications, radio signal measurements and emergency communications. 